\title{
Basic Structure Principle and Application of High Precision Microcomputer Automatic Calorimeter
}

\author{
Gang Li, Jian Chu \\ Tianjin Key Laboratory of Information Sensing and Intelligent Control, Tianjin University of Technology and \\ Education,Tianjin,300222,China
}

\begin{abstract}
This paper mainly expounds the system composition and the use of high precision microcomputer automatic calorimeter. Taking 80oA type LRY as an example, this paper introduces the main engine, oxygen bomb, automatic oxygen filling instrument, measurement and control software, computer, printer and other parts of the function and working principle and the problem should pay attention to in the use of the process.
\end{abstract}

Keywords: Microcomputer automatic calorimeter; oxygen bomb; automatic oxygen filling instrument.

\section{Introduction}

The system runs in Wnidow7 and above systems, human-computer interaction that is, learning to use. The soft using the object-oriented programming method, using the modular management technology, multi task operation. The system use advanced serial communication technology, integration of system control and data management, owning good compatibility and easy to maintain. It overcomes the disadvantages of the computer interface board job hopping and has a wide adaptability. Because of the use of scientific and effective algorithm, the data accuracy is high, the system is stable and reliable. The microcomputer automatic calorimeter is mainly used in electric power, coal, paper, petrochemical, cement, agriculture, medicine, scientific research, teaching industry, or to determine the calorific value of coal, petroleum, chemical, food, wood, explosives and other solid or liquid fuel. The system can automatically complete the calibration of thermal capacity of the system and the determination of the heat of matter. The calibration of thermal capacity of the system adopts multi - dimensional selection method, and is flexible in real time. The measuring process and data processing of the heat of matter are automatically completed by the microcomputer, according to the content of sulfur, water and hydrogen, automatically transform the barrel calorific value, high calorific value, low calorific value. The measurement process uses the digital prompt and the image method, the image is visual.

1.The working principle of a microcomputer automatic calorimeter

Taking the calorific value of coal as an example, the calorific value of coal is measured in oxygen bomb calorimeter. A certain amount of oxygen bomb in the analysis of the sample, burning in the oxygen bomb that filled with full amount of oxygen. The heat capacity of the meter is determined by burning a certain amount of standard substance benzoic acid under similar conditions. According to the temperature rise of the test sample before and after the thermal system, and the ignition heat and other attached heating for correction, the test sample can be obtained heat. The heat of nitric acid formation and thermal sulfur correction (sulfuric acid and sulfur dioxide form the difference between the hot) is deducted from barrel and then get a high calorific value. After correction of the moisture content of coal (the original water and hydrogen generated from coal combustion), the heat of coal was obtained.

\section{The Composition of Microcomputer Automatic Calorimeter}

\subsection{Host}

Automatic water injection, drainage, will not overflow, water temperature is not required. The system using scientific and effective algorithm, which can automatically fix correction constant. It has the characters of high data accuracy, the system is stable and reliable, and data processing can be carried out after the experiment. It using Serial communication technology, the failure rate is low and the requirements of using environment is loose. Before the host water injection, should be carefully check: whether there is a foreign body inside the tube, all the water pump inlet and outlet pipe whether in place. the inner cylinder; water pump over whether all the import plug in place, there is no deformation condition, if the water outlet cap is tightened, whether the overflow gate is opened.(Tip:the water outlet and the water outlet joint is fixed at the lower right side of the back of the main engine.)

\subsection{Automatic oxygen filling apparatus}

The structure is simple, the operation is convenient, the gas sealing performance is good, and the sealing rubber ring is convenient to replace.

\subsubsection{Installation and inspection}

a) Before installation, carefully check whether the parts are tight, the appearance should be non destructive and collision phenomenon.

b) Connect the oxygen filling instrument through the oxygen filled catheter and the pressure reducing valve and the oxygen bottle, and tighten all the nuts.

c) Opening the total valve of the oxygen bottle, adjusting the pressure regulator on the screw, so that the low pressure gauge to $2.8 \mathrm{~A} 3 \mathrm{MPa}$ position, this time the whole gas pipeline should be no leakage phenomenon. Otherwise, reinstall until normal. When everything is 


\section{International Journal of Science and Research (IJSR) \\ ISSN (Online): 2319-7064 \\ Index Copernicus Value (2013): 6.14 | Impact Factor (2015): 6.391}

OK, the whole installation inspection work is over.

d) Oxygen bomb will be put into the oxygen filling apparatus to test oxygen, at this time should be no leakage and easy to operate, the pressure meter on the pressure indicator should be with the pressure relief valve on the low pressure indicator is basically the same.

\subsubsection{Using}

Opening valves for oxygen bottles, transferring low table indicating to 2.8 to $3 \mathrm{Mpa}$, putting the oxygen bomb on the oxygen filling device, so the oxygen warhead is aligned with the gas nozzle. Downward pressure oxygen filling handle, the pressure indication is 2.8 to $3.0 \mathrm{Mpa}$ (low pressure gauge). Oxygenation filling time is $30-45$ seconds, slowly release the handle after adequate oxygen. Taking out the oxygen bomb, this time should be able to see the valve core on oxygen bomb to highlight, said oxygen bomb has been filled with oxygen.

Matters needing attention:

a) The end of each day after the experiment, the oxygen valve should be closed, and the gas path in the oxygen releasing.(Method: closing the valve of oxygen valve , using oxygen bomb filling oxygen, until the oxygen pressure indicator is zero.)

b) The place of oxygen meter and oxygen bottle placed should be prohibited fireworks.

c) It is strictly prohibited to bend and twist the filling pipe.

d) Regularly check the indicator value between pressure gauge on the filling machine and the low pressure gauge on the pressure relief valve is consistent.

e) The connection point in the gas path can not be used with any grease.

f) After the oxygenation is finished, the handle of the oxygen filling instrument should be released slowly.

\subsection{Oxygen bomb}

\subsubsection{Checking before use of oxygen bomb}

Oxygen bomb has been carried out strict quality inspection before using, users do not have to disassemble, but the re-inspection of quality is essential.

a) Oxygen filled mouth with no loosening phenomenon.

b) Whether the two electrode rods are loose and the fire board is tighten.

c) Whether the crucible support is good.

d) Air inlet is smooth.

e) The screw and the sealing ring on the oxygen bomb tube and oxygen bomb cover are free of foreign matter.

f) There is no bubble to go up after oxygen bomb filled with oxygen and into the water.

g) Appearance with no collision trace.

\subsubsection{The notes of correctly using oxygen bomb.}

a) It is strictly prohibited to connect the two electrode of the fire blocking plate.

b) Every time before decorate the fire wire, the residual ignition rod and l other foreign body must be cleaned. c) It is strictly prohibited to fill oxygen overpressure, and oxygen filling time should be relatively consistent.

d) The oxygen bomb cover should not be too tight, spin in place after a little force.

e) Oxygen bomb must be regularly quality inspection, at least once a year to carry out water pressure test.

\section{System Debugging}

Open the automatic cylinder head : (1) Filling the water into the outside cylinder through the water injection port. (2) Through the inner tube, the water is injected through the inner cylinder in the way of drainage experiment. Running high precision microcomputer instrument system software, familiar with the interface of the information. The first to observe the system connection is normal, normal should show the environment temperature, the outer cylinder water began to stir. Then use the mouse to click and open the system test window, to detect whether the internal mixing and ignition system is normal. After the normal return to the main window, enter the login window to modify system parameters. Especially the injection time, we must set reasonable. Taking oxygen bomb into the inner cylinder, from small to large test injection time setting. If water injection time is too short, inner tube water is insufficient, or adjustment is not stable or can not be adjusted, it is difficult to accurately test. If water injection time is too long, the water may overflow from the inner cylinder. Don't leave the scene when debugging, pay attention to observe the surface. If there is an accident, please turn off the power supply. The system water injection time is generally 30 to 40 second range, which is according to local voltage situation. The observation experiment was carried out through the water injection. In addition, using a universal table for ignition detection, ignition time is generally 3 to 8 seconds, which is automatic controlled by the program.

\section{Daily maintenance and inspection}

After the end of each use, should always carry out the following inspection and maintenance, so it can make the instrument often maintain good working condition and can prolong the service life.

4.1 Oxygen bomb: in addition to clean and dry the oxygen bomb after each experiment, and the following points should also pay attention to and check.

a) Oxygen bomb can only be used by hand, when the hand fells tight should stop, do not by all tool. Every time the test is completed, should clean the oxygen bomb.

b) The elastic cap and valve seat should be washed and dried after each of use.

c) Play the cup clean, scrub the thread, and check the upper part of the cup with no mechanical damage, pay attention to don't make the cup upside down.

d) Check the sealing ring if there is wear and burn injuries, and if the seal is not strictly, it should be replaced.

e) Check the insulation is good, and there is no damage.

f) $20.0 \mathrm{Mpa}$ water pressure test is carried out on oxygen bomb at regular intervals, and the use time of oxygen 


\section{International Journal of Science and Research (IJSR) \\ ISSN (Online): 2319-7064 \\ Index Copernicus Value (2013): 6.14 | Impact Factor (2015): 6.391}

bomb is not more than one year (or no more than 5000 tests).

\subsection{The water jacket}

If not for the test, the water in the tube must be put off, keep clean inside, do not make the dirt fall into the barrel.

\section{Performance Characteristics}

The automatic calorimeter has the following characteristics: By using the compressor refrigeration mode, the temperature of the inner and outer tube is automatically adjusted, the cooling correction coefficient is reduced, and the measuring result is more accurate. It meet the national standard $\mathrm{GB} / \mathrm{T} 213-2008$ that the inner tube is about $1 \mathrm{~K}$ higher than the outer tube at the end. The microcomputer calorimeter maintains all the functions of the microcomputer system, which can be used for other transactions, automatic calibration of the thermal capacity of the system and the measurement of heat. When inputting sulfur, water, hydrogen and other data, you can convert and print out the cartridge heat, high calorific value, low heat and other data. The inner tube of the heat measuring instrument adopts the electric stirring of the paddle blade, and the outer drum is stirred by a submersible electric stirring, which makes the stirring is more uniform and more convenient. The instrument adopts the fuse type ignition mode. Microcomputer based calorimeter operating in WindwsXP and above operating system, human-computer interaction, that is to learn to use, according to the prompts to complete the test.

\section{References}

[1] Xue Wu, Taiping Zhang, Hanjiong Wu. The industrial analysis method of coal and the development of instrument for measuring instrument $[\mathrm{J}]$ Coal quality technology, 2008,5:19-22.

[2] Bin Zhou. Research and Discussion on the industrial analysis method of coal [J] Heilongjiang science and technology information.2012,3:12

[3] Guihong Zhang. An improved method for analysis of coal industry [J] Fuel and chemical engineering.2009,40 (01): 14-16

[4] Ying Zeng. Design of measurement and control system for high precision differential scanning calorimetry [D] Beijing: China Jiliang University, 2014

[5] Qingguo Wang, Ju Zhang, Feng Zhang. Working principle and application of cone calorimeter [J] modern scientific instrument.2003,06:36-39.

[6] Shuang Zhang, Liang Yang. An error analysis of the combustion heat value of the material with oxygen bomb calorimeter [J] Fire science and technology.2009,28 (7): 519-521.

[7] Lipeng Han. The accuracy of measuring the heat capacity of coal fired power plant [J] Coal quality technology, 2012,02:38-40.

[8] Biao Hu. Research and design of rapid calorimeter [D] Changsha: Hunan University, 2008
[9] Yanmin Wu, Lingyun Guo, Guoqiang Ding. Development of a new thermal insulation intelligent calorimeter. Application of electronic technology.2013,39 (11): 139-141

[10] Zhenghua Zhang. Establishment of high precision and constant temperature environment electrochemical heat system [D] Changsha: Central South University, 2008

[11] Hua Song. Research on intelligent temperature control technology of calorimeter [D] Tianjin: Tianjin University of Science and Technology, 2012

[12] Hongliang Sun. Study on data analysis and prediction of calorific value of coal quality [D] Jilin: Northeast Dianli University, 2014

[13] Long Wang,Xianhong Li, Yang Xu. Research status of coal quality analysis instrument and its measurement standard [J] Industrial measurement, 2012,02:158-160

[14] Yanan Wang. Research on error control in coal quality analysis [J] Science and technology information, 2015,18:216-217 